Kansas State University Libraries

New Prairie Press

\title{
A TRANSFORMATION APPROACH TO ESTIMATING USUAL INTAKE DISTRIBUTIONS
}

Sarah M. Nusser

Alicia L. Carriquiry

Helen $\mathrm{H}$. Jensen

Wayne A. Fuller

See next page for additional authors

Follow this and additional works at: https://newprairiepress.org/agstatconference

Part of the Agriculture Commons, and the Applied Statistics Commons

\section{(c) (1) $(9)$}

This work is licensed under a Creative Commons Attribution-Noncommercial-No Derivative Works 4.0 License.

\section{Recommended Citation}

Nusser, Sarah M.; Carriquiry, Alicia L.; Jensen, Helen H.; and Fuller, Wayne A. (1990). "A TRANSFORMATION APPROACH TO ESTIMATING USUAL INTAKE DISTRIBUTIONS," Conference on Applied Statistics in Agriculture. https://doi.org/10.4148/2475-7772.1438

This is brought to you for free and open access by the Conferences at New Prairie Press. It has been accepted for inclusion in Conference on Applied Statistics in Agriculture by an authorized administrator of New Prairie Press. For more information, please contact cads@k-state.edu. 


\section{Author Information}

Sarah M. Nusser, Alicia L. Carriquiry, Helen H. Jensen, and Wayne A. Fuller 


\title{
A TRANSFORMATION APPROACH TO ESTIMATING USUAL INTAKE DISTRIBUTIONS
}

\author{
Sarah M. Nusser, The Procter and Gamble Company* \\ Alicia L. Carriquiry, \\ Helen H. Jensen, and Wayne A. Fuller \\ Iowa State University ${ }^{* *}$
}

\begin{abstract}
Design of effective food and nutrition policies, efficient allocation of resources, and more precise targeting of food programs require good estimates of the percentage of the population with deficient, or excess, nutrient or other food component intake. An individual's mean daily intake of the dietary component is a good estimate of the individual's dietary status. However, to evaluate dietary adequacy of a population it is necessary to obtain an estimate of the distribution of usual intakes. Often, the distribution of usual intakes is estimated from the distribution of mean daily intakes. Two problems arise. First, distributions of usual intakes for most nutrients are skewed. Second, the variance of the distribution of mean daily intakes is larger than the variance of the true usual intake distribution, due to within-individual variability of daily intakes. We describe a method for estimating usual intake distributions which does not assume normality, and which takes into account the within-individual variation in daily intake. The method relies on appropriate transformation of the dietary data from the original space into normal space.
\end{abstract}

\section{Introduction}

The U.S. Department of Agriculture (USDA) has collected data on the food consumption patterns of households since the mid 1930s. Initially designed to assess the adequacy of food supplies, the data currently support a wide range of government monitoring and programmatic functions. The data also provide basic information for understanding food behaviors in order to develop effective food and agricultural policies. By means of extensive food composition data banks, USDA uses data collected on the basis of food intakes to evaluate consumption. of nutrients and other dietary components. The estimated levels of consumption provide information on trends in the U.S. diet as well as on factors that determine problems of food consumption and nutrient intake.

Assessing the nutritional status of a population is important for the formulation and targeting of food assistance programs, consumer education, and for food regulatory activities. Dietary data provide one source of information for assessing nutrient adequacy. The most common diet-based

\footnotetext{
*Wile this research was being conducted, S. M. Nusser was at Iowa State University.

${ }^{* *}$ Financial support for this research was provided by the Human Nutrition Information Service, USDA, Research Support Agreement No. 58-3198-9-032.
} 
measure of nutritional status is the usual daily nutrient intake of an individual. The usual daily intake of a nutrient is defined to be the normal or long-run average intake of the nutrient for a given individual. Use of usual intakes as a measure of nutritional status explicitly recognizes that a low intake of the dietary component on one day is not an indicator of a nutritional deficiency; rather, it is insufficient intake over a long period of time that places an individual at risk for nutritional deficiency (NRC, 1989).

Usual intakes are generally unobservable, but are estimated typically from data on daily intakes collected from individuals for a number of days. An individual's usual intake of a dietary component is often estimated by the individual's mean daily intake of the dietary component. While this methodology is appropriate for assessing an individual's usual nutrient intake, a different approach is required to evaluate dietary adequacy of a population (NRC, 1986; Ritenbaugh et al., 1988; Nusser et al., 1990). In the latter case, it is necessary to obtain an estimate for the distribution of usual intakes.

Often, the distribution of usual intakes is estimated from the distribution of mean daily intakes. Further, it is frequently assumed that usual intakes of nutrients are normally distributed. This approach gives rise to two problems. First, the distribution of usual intakes of most dietary components is right-skewed, and therefore, not normal (Jensen et al., 1989; Nusser et al.: 1990). Second, the within-individual variability of daily intakes causes the variance of the distribution of mean daily intakes to be larger than that of the true usual intake distribution (Ritenbaugh et al., 1988; Jensen et al, 1990). Because the tails of the empirical mean distribution are too large relative to the usual intake distribution, prevalence of a dietary deficiency (i.e., the proportion of the population whose intakes fall below a specified level) is overestimated. Overestimation increases as the number of days of intakes observed for an individual declines. One solution to the problem of overdispersion in the estimated distribution was suggested by the National Research Council (1986), and consisted of adjusting the individual means so that their variance is the same as the estimated variance of usual intakes. This approach produces the correct estimate of the usual intake under normality. For other distributions, it scales the data so that the estimated usual intake distribution has the proper mean and variance, but does not adjust other attributes of the distribution, such as skewness.

In what follows, we describe an alternative method for estimating usual intake distributions which does not assume normality, and which takes into account the presence of within-individual variation of daily intakes. The approach we suggest consists of transforming the dietary data from the original scale into normality, and predicting the usual daily intakes in normal space. These predicted usual intakes can then be retransformed to the original scale, to obtain a set of pseudo usual intakes. In Section 2, we describe general attributes of the data used for estimating usual intake distributions and a specific data set used to develop our approach. The proposed methodology is given in Section 3. An application to two dietary components, energy and vitamin $C$, is presented in Section 4.

\section{Dietary Intake Data}

2.1 Description of the Data Data suitable for estimating usual intake distributions of dietary 
components should allow for the estimation of between- and within-individual variances. The within-individual variance can be obtained as long as the data set includes more than one day of intake data on each individual. Intake data on individuals allow for the estimation of between-individual variance. Use of one day intake data is not appropriate for estimation of usual intake distributions of nutrients. These 1 -day intake data sets do not provide a means for distinguishing the within-from the between-individual variances.

Data from the Continuing Survey of Food Intakes by Individuals (CSFII) were used to help develop the methodology described in section 3 . The CSFII data were collected by the Human Nutrition Information Service (HNIS) of the USDA in 1985-1986. Daily dietary intakes were collected from women between 19 and 50 years of age and from their preschool children. Daily intakes were to be obtained at approximate two-month intervals over the period of one year (April 1985 to March 1986). Data for the first day were collected by personal interview and were based on a 24-hour recall. Data for subsequent days were based on 24-hour recall and were collected by telephone whenever possible. The sample was a multi-stage stratified area probability sample from the 48 coterminous states. The primary sampling units were area segments, and the probabilities of selection of area segments were proportional to the numbers of housing units in the segments as estimated by the Bureau of the Census. Because of the high rate of nonresponse for the six-day sample, the USDA constructed a four-day data set for analyses. The four days consisted of the first day of dietary intakes for all individuals who provided at least four days of data, plus a random selection of three daily intakes from the remaining three, four or five days of data available. A subset of the CSFII four-day data set corresponding to 23-50 year-old women who were not pregnant or lactating was used in the analysis below. USDA (1987) converted data on food intakes for each individual into respective nutrient and other dietary components using extensive food composition data banks. Dietary components under consideration were calcium, energy, iron, protein, vitamin $A$, and vitamin C. In this paper, we focus on the results for energy and vitamin $C$, which have very different intake distributions.

\subsection{Results from Preliminary Analyses}

The daily intake data were examined using analysis of variance methods to determine whether weekday, month, interview method (personal or telephone) and interview sequence effects were important. Interview sequence refers to the order in which the daily data were obtained for sample individuals; there were four values corresponding to this variable. Weekday effects were significant for energy $(p<0.001)$ and protein $(p<0.01)$ intakes. Sequence effects, confounded with month effects, were significant at the 0.001 level for calcium, energy, iron and protein intakes. Therefore, the data used in subsequent analysis were adjusted by interview sequence and weekday effects. A ratio adjustment, rather than the usual linear adjustment, was used to insure that all adjusted values were nonnegative.

The inter- as well as the intra-individual variance for observed daily intake was estimated for each nutrient. Results indicated that the intra-individual variance of the daily intakes for the dietary components involved in the study was about twice as large as the inter-individual variance. These results are in agreement with those reported by sempos et a1. (1985).

Basic features of the distributions of four-day average intakes of the dietary components were obtained by calculating estimates for the mean, 
standard deviation, skewness and kurtosis (SAS, 1985, pp. 737-741). Skewness estimates indicated that the distributions of the four-day average intakes were skewed to the right. The energy and protein intakes were the least skewed.

The sample variance of the daily intake for each individual in the sample was computed, and its square root was plotted against the corresponding individual average daily intake. The plots we obtained suggested that the true standard deviation of individual intakes may be directly proportional to the usual intake of the individual. Similarly, plots of the cube root of the intra-individual third moments against average daily intakes suggested that the cube root of the third moment of the individual daily intakes may be a linear function of the usual intake.

\section{The Transformation Approach}

\section{1 overview}

A nonparametric approach to transforming the data is described below. The objective of this approach is to produce transformed observations that are normally distributed and have homogeneous variances.

The approach we suggest for estimating the distribution of usual intakes of a nutrient involves the following steps: 1) observed intakes are transformed to normality, 2) the normal data are assumed to follow a measurement error model that decomposes the observed daily intake of an individual into the usual intake for that individual plus a measurement error associated with the individual on the day the intake was observed,

3) normal theory is then used to obtain predictors of usual intakes in normal space for each individual, 4) application of an inverse transformation to the predicted normal usual intakes produces a set of pseudo usual intakes in the original scale, which can then be used to estimate the distribution of usual intakes. The measurement error model approach requires an estimate of the within-individual variation, which can be obtained only if data for each individual are available for more than one day.

\subsection{Transforming the Observed Data to Normality}

The transformation of the observed data to normality consists of the following: first, a smoothed empirical cumulative distribution function of the observed daily intakes is evaluated at each of these values to produce a set of uniform random variables. The inverse normal cumulative distribution function is then used to transform the uniform variates into a set of standard normal random variables.

Let $Y_{k, j}$ denote the observed intake of a dietary component $k$ for individual 1 on day $j$, where $k=1, \ldots, p$ components, $i=1, \ldots, n$ individuals, and $j=1, \ldots, r$ days. Assume that individuals, as well as daily intakes within individuals, are independent. The empirical cumulative distribution function constructed from the $n r Y_{k i j}$ values is a step function. By connecting the midpoints of the rises between the steps defined by the empirical c.d.f., a continuous piecewise linear estimate of the true cumulative distribution function $F_{Y k}$ is constructed. For this choice of midpoints, the continuous cumulative distribution function yields approximately the same mean value of the data as the empirical cumulative distribution function.

The estimated continuous c.d.f. provides a means of generating a set of uniform $(0,1)$ variates, $P_{k i j}$, from the observed intakes. Therefore, given the standard normal cumulative distribution function $\Phi($.$) ,$ 


$$
z_{k i j}=\Phi^{-1}\left(p_{k i j}\right)
$$

are $N(0,1)$ variates. The $X_{t i j}$ represent the transformed observed values. It may be the case that the transformed values do not have homogeneous within-individual variances. If so, a further transformation is required to homogenize the within-individual variances.

\subsection{An Approximation to Multivariate Normality}

The transformation procedure described in Section 3.2 produces a set of $N(0,1)$ variables, but the transformed intakes of the $p$ dietary components will not necessarily be jointly normal.

Should the departure from multivariate normality be pronounced, it is possible to further transform the data to approximate multivariate normality. First, a set of uncorrelated linear combinations of the dietary components are obtained from:

$$
\mathbf{X}_{\mathbf{j}}=\mathbf{T} \mathbf{X}_{i j},
$$

where $T$ is a linear transformation matrix derived from a measurement error model assumed on the $\boldsymbol{X}_{\mathrm{ij}}$. The elements of $\mathbf{X}_{\mathrm{ij}}^{*}$ are uncorrelated random variables, but are not necessarily multivariate normal. Then, letting $\Phi^{-1}($. represent the inverse of the normal cumulative distribution function, for each dietary component. $\mathrm{k}$,

$$
Z_{k i j}=\Phi^{-1}\left(X k_{i j}\right),
$$

where the elements of $z_{i j}$ are marginally normal, nearly uncorrelated random variables. It seems reasonable to assume that the elements of $z_{i j}$ are approximately multivariate normal.

\subsection{Predicting Usual Intakes in Normal Space}

Normal theory and a measurement error model can be used to generate predicted usual intakes from the transformed observed intakes. the prediction methodology is well suited for application to a vector of dietary components. The multivariate approach permits incorporation of information contained in the relationships among intake patterns of dietary components into the prediction of normal usual intakes.

Assume that data are available for $p$ dietary components on each individual. Suppose that for each dietary component $k$, the $n r$ values of $Y_{k j j}$ are transformed, using the methodology in Section 3.2 , to generate $n r X_{4 i j}$ normally distributed values. Denote the $p \times 1$ vector of transformed observations for individual $i$ on day $j$ by $Z_{i j}$.

A measurement error model is used as a basis for predicting the usual intakes given the observed intakes. Let

$$
\begin{aligned}
& z_{i j}=z_{i}+u_{i j} \\
& z_{i}-N_{p}\left(\mu_{x}, \Sigma_{2 x}\right)
\end{aligned}
$$




$$
\mathbf{u}_{i j}-\mathbf{N}_{p}\left(0, \Sigma_{u u}\right)
$$

where $x_{i}$ is the vector of unobservable usual intakes for individual $i$; $u_{i j}$ is the unobservable measurement error for individual $i$ on day $j$; the $x_{i}$ are independently distributed; the $u_{i j}$ are independent across days; and $\boldsymbol{z}_{i}$ and $\boldsymbol{u}_{i j}$ are uncorrelated. Assume that $\Sigma_{\text {w }}$ and $\Sigma_{w}$ are positive definite. This model implies that the $X_{i j}$ are $N\left(\mu_{x}, \Sigma_{x x}+\Sigma_{w u}\right.$ variates, and that the sample individual means

$$
\Sigma_{\mathrm{XX}}=\Sigma_{\mathrm{zx}}+\mathrm{I}^{-1} \Sigma_{\mathrm{uu}}
$$

are independent random variables from a $N\left(\mu_{x}, \Sigma \overrightarrow{X X}\right.$ distribution, with

$$
\Sigma_{\overline{X X}}=\Sigma_{x x}+r^{-1} \Sigma_{u u}
$$

It should be noted that if the normal observed intakes from the initial transformation described in section 3.2 are used in this model, $\mu_{x}=0$. However, $\mu_{x}$ may be nonzero if further transformations are required to obtain homogeneous error variances for the transformed intakes.

Our objective is to produce a set of pseudo usual intakes whose distribution is close to that of true usual intakes. The best linear unbiased predictor of $x_{i}$ (BLUP) has smallest prediction error variance among all unbiased linear predictors, and so would be appropriate if the objective was to predict individual $x_{i}$. However, if the BLUP is used to predict a set of $x_{i}$, the variance of the predicted $x_{i}$ is smaller than $\Sigma_{\alpha_{x}}$. Predictors of $x_{i}$ with unconditional variance $\Sigma_{\alpha x}$ can be obtained by using

$$
x_{i}^{*}=\mu_{x}+\Sigma z x^{2} \Sigma \frac{1 / 2}{X X}\left(\bar{X}_{1}-\mu_{x}\right)
$$

An analogous adjustment for empirical Bayes estimation was suggested by Louis (1984) when the objective of prediction is to obtain estimates whose empirical cumulative distribution function is close to the true cumulative distribution function. The values of $\mu_{x}, \Sigma_{x}$ and $\Sigma \bar{x}$ re unknown.

Therefore, to implement the procedure of [4], estimates of $\mu_{x}, \Sigma_{z x}$ and $\Sigma \overline{\mathrm{XX}}$ can be substituted into [4] in the appropriate places.

Usually, inferences are made about the dietary status of the target population regarding a single nutrient. It may be of interest, however, to assess dietary status with respect to a vector of nutrients. These predicted normal usual intakes can be used to make inferences about dietary status of the target population in multivariate normal scale. For example, suppose that we want to know the proportion of the population deficient for all $p$ nutrients, where deficiency is indicated by usual intakes below a vector $k$ of $p$ dietary requirements. In normal space, this proportion is given by $\operatorname{Pr}\left\{\boldsymbol{x}_{\mathbf{i}}<\mathbf{k}^{*}\right\}$, where the $\mathbf{x}_{i}^{*}$ are obtained from either the $\mathbf{x}_{i j}$ or the $z_{i j}$, and $k^{*}$ is the transformed vector $k$. Alternatively, predicted normal usual intakes can be transformed back to the original scale using the transformation described in section 3.5, and inferences can then be made from usual intake distributions estimated in the original scale. 


\subsection{The Mean Transformation}

The predicted usual intakes in normal space can be transformed to obtain a set of pseudo usual intakes in the original space. To generate a set of pseudo usual intakes in the original data scale from the normal usual intakes, a transformation from the normal space to the original scale is required. This transformation, called the mean transformation, should have the property that the usual intake in the original scale is equal to the mean transformation of the normal usual intake. Note that since the transformation from observed intakes to normal observed intakes is nonlinear, the inverse of this transformation cannot be used to transform normal predicted usual intakes (which are like means) back to the original scale. The remainder of the discussion on the transformation on $\mathrm{X}^{i j}=\mathrm{T}^{-1} \mathrm{Z}_{\text {ij }}$ is addressed for a single dietary component.

Let $\mathrm{g}_{\mathrm{k}}$ represent the transformation which carries the observed intakes for dietary component $\mathrm{k}$ into normality, i.e.,

$$
x_{k i j}=g_{k}\left(Y_{k i j}\right) \text {. }
$$

Let $h_{k}$ represent the inverse of this transformation so that

$$
Y_{k i j}=h_{k}\left(x_{k i j}\right)
$$

The transformation $h_{k}$ may be complicated and may not be explicitly expressed. A Taylor series approximation for $h_{k}\left(x_{k i}+u_{k i j}\right)$, where $x_{k i}$ and $u_{k i j}$ are the $k$-th elements in $x_{i}$ and $u_{i j}$ can be used to estimate $h_{k}\left(x_{k i j}\right)$. This approximation requires expressions for $h_{k}\left(x_{k i}\right)$ and for $h_{k} n\left(x_{k i}\right)$.

Preliminary analyses indicate that $h_{k}\left(x_{k i}\right)$ can be estimated from ( $k_{k i j}$, $\left.Y_{k i j}\right)$ pairs, via a grafted polynomial function with linear end segments and quadratic interior segments. Further, the second derivative $h_{k}$ " can be locally approximated for each individual by fitting a simple quadratic function to the estimated $h_{k}$. These estimators can then be used to construct the mean transformation which generates pseudo usual intakes $y_{k i}$ as follows:

$$
y_{k i}^{*}=\hat{h}_{k}\left(x_{k i}^{*}\right)+2^{-1} \hat{h}_{k}^{\prime \prime}\left(x_{k i}^{*}\right) \hat{\sigma}_{u u k k}
$$

where $\hat{h}_{k}$ and $\hat{h}_{k}^{\prime \prime}$ are the estimates of $h_{k}$ and $h_{k}^{\prime \prime}$ respectively, and $\hat{\sigma}_{\text {uukk }}$ is the $k$ - th element of $\Sigma_{u u}$.

\section{Application to the CSFII Data}

The procedures given in the previous Section were applied to the subset of the 1985-86 CSFII data described in Section 2. Four independent days of intake were available for each of 785 women, so that there were 3,140 observed intakes for each of 6 evaluated dietary components. Here, we present results for energy and vitamin $C$.

The observed intakes for each dietary component were transformed to normality using the procedure described above. The methods described in Section 3.4 for predicting normal usual intakes assume that the variance of the errors is homogeneous for each dietary component. To check the validity of this assumption for the CSFII data, for each dietary component $k$, plots were constructed of the standard deviation for individual $i, S_{k i}$, against the mean for individual $i, \bar{x}_{k i}$, where 


$$
\begin{aligned}
\bar{x}_{k i} & =r^{-1} \sum_{j=1}^{r} x_{k i j} \\
s_{k i}^{2} & =(r-1)^{-1} \sum_{j=1}^{r}\left(x_{k i j}-\bar{x}_{k i}\right)^{2} .
\end{aligned}
$$

These plots are shown for the two dietary components in Figures 1 and 2. The plots suggest that the assumption of homogeneous error variances for each dietary component is reasonable.

The assumption that the vectors of dietary component means for each individual follow a multivariate normal distribution was also investigated. A chi-square test for univariate normality was performed on the individual means of the transformed intakes. The test indicated that the marginal distribution of the means does not significantly differ from a normal distribution for any dietary component. Tests for bivariate normality were constructed in a similar fashion, for pairs of observed means of dietary components. Results indicated that not all dietary component pairs have a distribution consistent with bivariate normality. These tests suggest that the assumption of multivariate normality for a vector of dietary component means is probably not true. However, plots showed that the departures do not appear to be severe enough to warrant development of an alternative prediction method. Energy and vitamin $C$ were among the pairs of dietary components which exhibited a bivariate normal distribution.

The estimated covariance matrices for within individual variation, $\Sigma_{u u}$, and among individual variation, $\hat{\Sigma}_{\overline{X X}}$, obtained in the prediction process, are listed in Table 1 along with the estimated covariance matrix of the normal usual intakes, $\hat{\Sigma}_{\mathrm{Xx}}$, where
$\hat{\Sigma}_{\mathrm{Xx}}=\hat{\Sigma}_{\overline{\mathrm{XX}}}-0.25 \hat{\Sigma}_{\mathrm{uu}}$.

To develop the mean transformation for transforming normal usual intakes to the original scale, a segmented polynomial approach was used to estimate $h_{k}$ for each dietary component from $\left(Y_{k i j}, X_{k i j}\right)$ pairs. The first 10 and last 10 observations were fit with linear segments. Ten inner segments of equal length were created from the remaining 765 observations. Each inner segment was modeled with a quadratic function. The regression model was restricted, so that each segment joined smoothly to create a continuous curve with a continuous first derivative. Plots of the fitted transformation $h_{k}$ and the original obșerved intake pairs $\left(Y_{k i j}, X_{k i j}\right)$ are presented in Figures 3 and 4.

Using $h_{k}$ and the approximation for $h_{k}{ }^{n}$ described in Section 3.5 , the mean transformation was constructed and the normal usual intakes were transformed back to the original scale to create a set of pseudo usual intakes.

Relative frequency histograms depicting the distribution of pseudo usual intakes for each dietary component are presented in Figures 5 and 6 . Each distribution has some degree of right-skewness. This is particularly true for vitamin $C$. In the case of energy, a shift in the origin from zero to a positive value may be useful in fitting parametric distributions to the data; as expected, this plot indicates that usual intakes for energy are unlikely to be very close to zero. 


\section{References}

Jensen, H. H., S. M. Nusser, W. A. Fuller, and S. R. Johnson. 1989. Estimating the distribution of usual nutrient intake. Paper prepared for presentation at the AIN Fall Meeting on Nutrition Monitoring and Nutrition Status Assessment, Charleston, S. C., December 8-10, 1989.

Jensen, H. H., S. M. Nusser, and L. Sands. 1990. Methods for assessment of the nutrient adequacy of diets. Unpublished manuscript.

Louis, T. A. 1984. Estimating a population of parameter values using Bayes and empirical Bayes methods. Journal of the American Statistical Association 47:393-398.

National Research Council. 1986. Nutrient Adequacy: Assessment Using Food Consumption Surveys. National Academy Press, Washington, D. C., 146 p.

National Research Council. 1989. Recommended Dietary Allowances. National Academy Press, Washington, D. C., 284 p.

Nusser, S. M., G. E. Battese, and W. A. Fuller. 1990. Method of moments estimation of usual nutrient intake distributions. CARD Working paper 90-WP 52, Iowa State University, Ames.

Nusser, S. M., and W. A. Fuller. 1990. A transformation approach to generating pseudo usual intakes. Preliminary progress report for the Human Nutrition Information Service, USDA, for Research Support Agreement $58-3198-9-032$.

Ritenbaugh, C., G. Beaton, C. S. Goodby, C. Feldman, and M. Aickin. 1988. Methodological issues in food consumption surveys. Final report for USDA Cooperative Agreement 非 58-3198-6-62.

Sempos, C. T., N. E. Johnson, E. L. Smith, and C. Gilligan. 1985. Effects of intraindividual and interindiviudal variation in repeated dietary records. American Journal of Epidemiology 121:120-130.

U.S. Department of Agriculture, Human Nutrition Information Service, Nutrition Monitoring Division. 1987. Women 19-50 years and their children, $1-5$ years, 4 days. Nationwide Food Consumption Survey Continuing Survey of Food Intakes by Individuals. NFCS, CSFII: Report No. 85-4. 


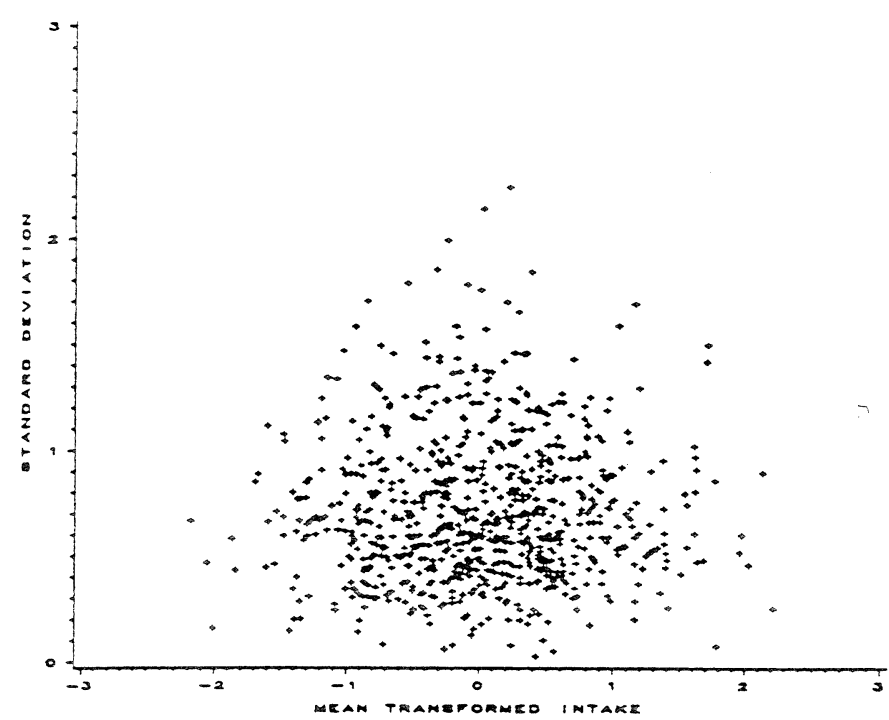

Figure 1. Plot of individual standard deviations against individual means calculated from transformed data for energy.

Source: CSFII, 1985-1986.

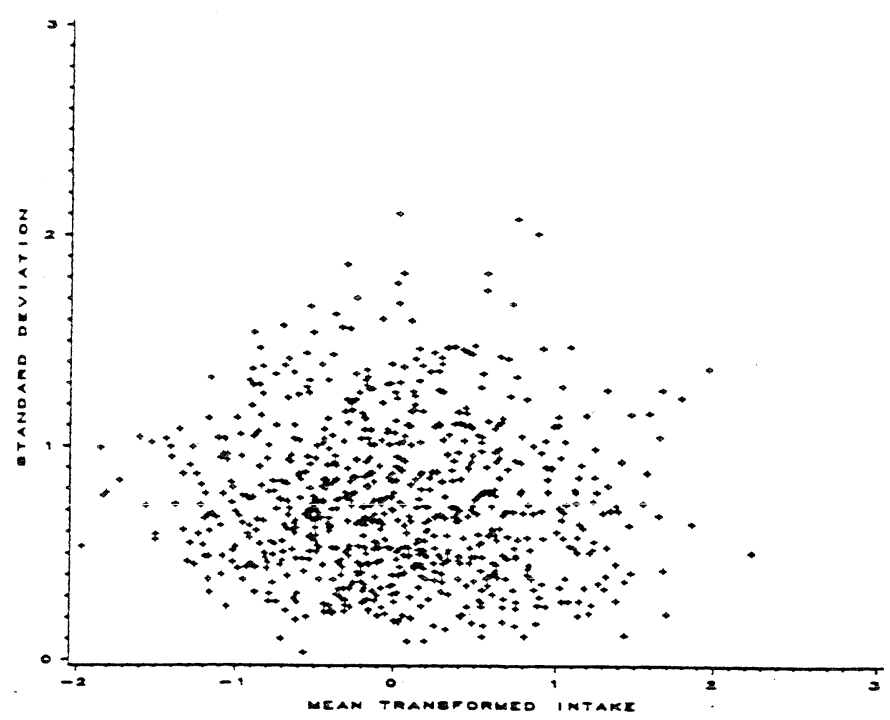

Figure 2. Plot of individual standard deviations against individual means calculated from transformed data for vitamin C.

Source: CSFII, 1985-1986. 


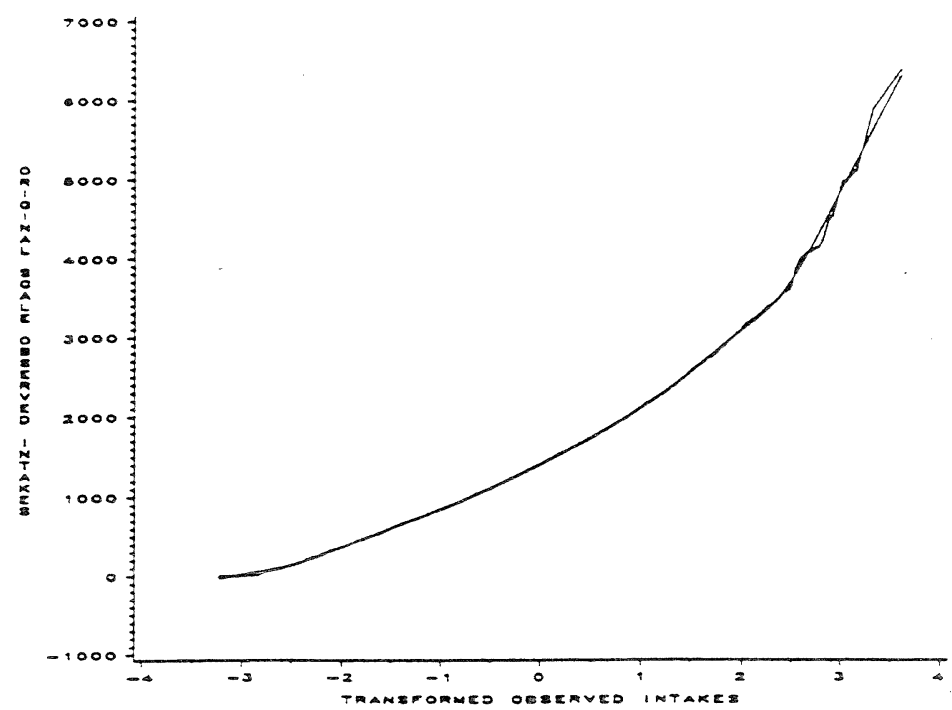

Figure 3. Plot of the fitted inverse transformation $\hat{h}_{k}$ (smooth line) and the observed intake data $\left(Y_{k i j}, X_{k i j}\right)$ for energy.

Source: CSFII, 1985-1986.

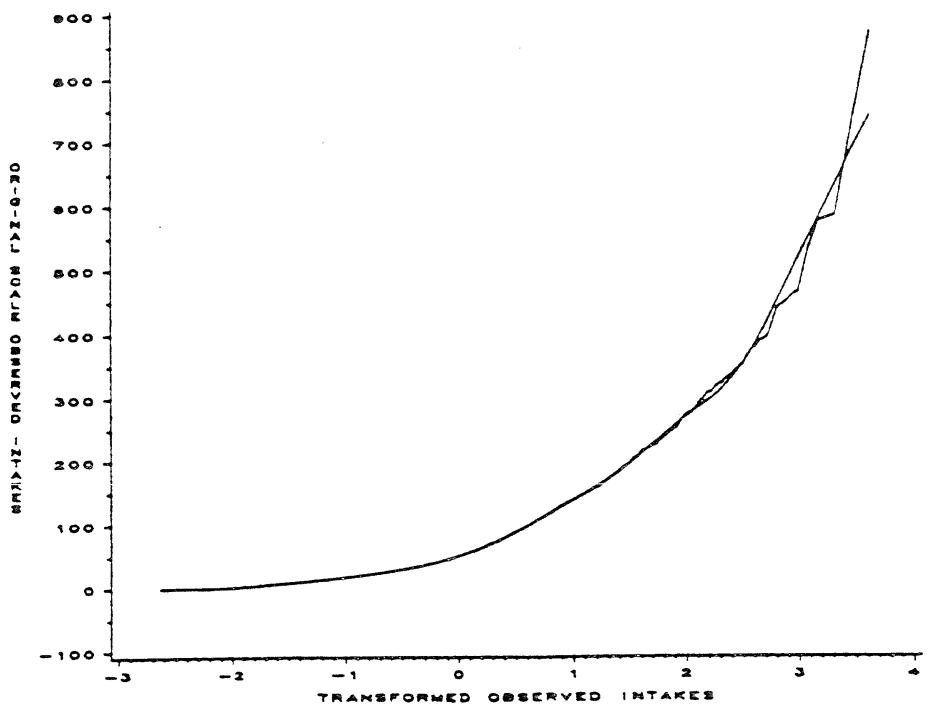

Figure 4. Plot of the fitted inverse transformation $\hat{h}_{k}$ (smooth line) and the observed intake data $\left(\mathrm{Y}_{\mathrm{kij}}, \mathrm{X}_{\mathrm{kij}}\right.$ ) for vitamin $\mathrm{C}$. Source: CSFII, 1985-1986. 


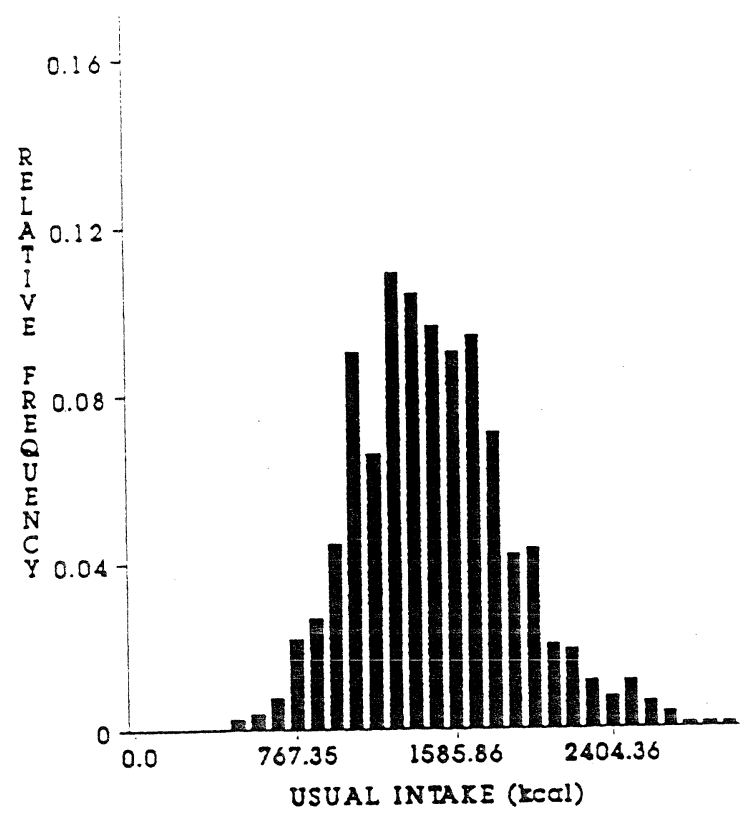

Figure 5. Relative frequency histogram of the pseudo usual intake distribution for energy.

Source: CSFII, 1985-1986.

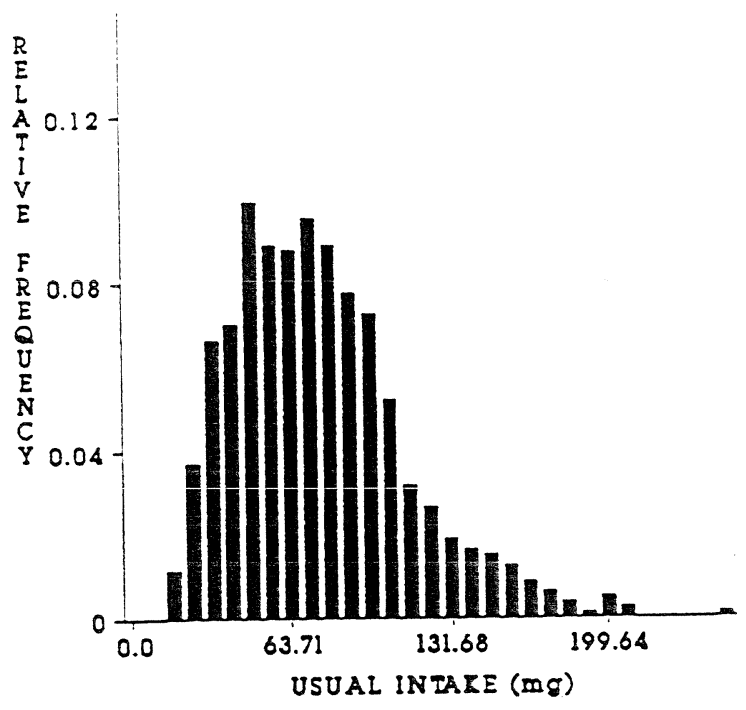

Figure 6. Relative frequency histogram of the pseudo usual intake distribution for vitamin $C$. Source: CSFII, 1985-1986. 
Table 1. Estimated covariance matrices for intra-individual variation $\left(\Sigma_{u u}\right)$, inter-individual variation $\left(\Sigma_{\overline{\mathrm{XX}}}\right)$, and normal usual intakes $\left(\Sigma_{\Sigma x}\right)$

\begin{tabular}{|c|c|c|c|c|c|c|}
\hline $\begin{array}{c}\text { Covariance } \\
\text { matrix }\end{array}$ & Calcium & Energy & Iron & Protein & Vit. A & Vit. C \\
\hline$\Sigma_{\text {uu }}$ & .6380 & $\begin{array}{l}.3701 \\
.6302\end{array}$ & $\begin{array}{l}.3031 \\
.4560 \\
.6827\end{array}$ & $\begin{array}{l}.3452 \\
.4954 \\
.5003 \\
.7246\end{array}$ & $\begin{array}{l}.2685 \\
.2384 \\
.2937 \\
.2456 \\
.7699\end{array}$ & $\begin{array}{l}.1780 \\
.2063 \\
.2121 \\
.1751 \\
.2828 \\
.6846\end{array}$ \\
\hline$\hat{\Sigma_{\overline{X X}}}$ & .5213 & $\begin{array}{l}.3751 \\
.5271\end{array}$ & $\begin{array}{l}.3201 \\
.3916 \\
.4871\end{array}$ & $\begin{array}{l}.3362 \\
.3974 \\
.3802 \\
.4550\end{array}$ & $\begin{array}{l}.2770 \\
.2180 \\
.2813 \\
.2238 \\
.4185\end{array}$ & $\begin{array}{l}.2087 \\
.2047 \\
.2542 \\
.2085 \\
.2735 \\
.4822\end{array}$ \\
\hline$\hat{\Sigma}_{\mathrm{xx}}$ & .3618 & $\begin{array}{l}.2825 \\
.3639\end{array}$ & $\begin{array}{l}.2443 \\
.2776 \\
.3164\end{array}$ & $\begin{array}{l}.2499 \\
.2736 \\
.2551 \\
.2739\end{array}$ & $\begin{array}{l}.2098 \\
.1584 \\
.2079 \\
.1624 \\
.2260\end{array}$ & $\begin{array}{l}.1642 \\
.1532 \\
.2012 \\
.1648 \\
.2028 \\
.3111\end{array}$ \\
\hline
\end{tabular}

Source: CSFII, 1985-1986. 